\title{
Mini tunelizador moldável: um avanço técnico no acesso vascular para hemodiálise
}

\author{
Mini moldable tunneler: a technical improvement for hemodialysis access \\ Fabio Henrique Rossi', Nilo Mitsuru Izukawa², Akash Kuzhiparambil Prakasan³, Gisleno Serafim Lustosa, Patrik Bastos \\ Metzger ${ }^{4}$, Fernanda Maria Angeliere ${ }^{4}$, Eduardo da Silva Jordão ${ }^{4}$, Bruno Lourenço de Almeida ${ }^{4}$
}

\begin{abstract}
Resumo
Contexto: A confecção de fístula para hemodiálise pode envolver a criação de túnel para a acomodação do enxerto - veia autóloga ou prótese sintética. O trajeto e a extensão dependem do local escolhido para a realização das anastomoses e posicionamento do trajeto do enxerto. Na atualidade, os tunelizadores utilizados são constituídos de estruturas cilíndricas metálicas rígidas, que tornam necessário o uso de múltiplos tamanhos e conformações.

Objetivo: Testar a utilização de um único mini tunelizador universal metálico e moldável na cirurgia de confecção de fístula para hemodiálise. Métodos: Trata-se de um estudo piloto. O tunelizador desenvolvido consiste de uma estrutura cilíndrica e modular composta de fio de aço inoxidável moldável interno, com manopla em extremidade proximal e dupla ponta cônica intercambiável em extremidade distal e bainha cilíndrica externa de polietileno. O tunelizador foi utilizado em dez cirurgias de confecção de fístulas para hemodiálise em membros superiores e inferiores, e o sucesso de sua aplicabilidade e a frequência de complicações precoces foram observados.

Resultados: Suas características de maleabilidade e conformibilidade permitiram a utilização do mesmo tunelizador nos diversos tipos de fístulas realizadas. A ponta cônica distal com duplo diâmetro, intercambiável, permitiu que o mesmo aparato fosse aplicado na utilização de veia autógena ou prótese sintética. Todas as cirurgias foram seguidas de sucesso e com baixos índices de complicações precoces.

Conclusão: A versatilidade do mini tunelizador metálico maleável e moldável permitiu sua utilização em todas as fístulas realizadas, independentemente do sítio das anastomoses e o trajeto de acomodação escolhido, sem complicações e com excelente índice de perviedade. Os dados sugerem que o mini tunelizador metálico maleável moldável pode ser utilizado com segurança e vantagens nas cirurgias de confecção de fístula para hemodiálise. Sua versatilidade e baixo custo de produção podem levá-lo a substituir os tunelizadores rígidos utilizados na atualidade e apontam para estudos mais elaborados e com casuística adequada para avaliação do real valor do novo instrumento.
\end{abstract}

Palavras-chave: fístula arteriovenosa; diálise renal; cirurgia.

\footnotetext{
Abstract and conformations may be necessary.

Objective: Testing the use of a mini universal moldable tunnel maker in hemodialysis access fistula surgical creation. complications were studied during ten hemodialysis fistula creation. creation. complications and with excellent patency rate.

Keywords: fistula; renal dialysis; surgery.

Trabalho realizado no Departamento de Cirurgia Vascular do Instituto Dante Pazzanese de Cardiologia - São Paulo (SP), Brasil.

1 Doutor; Cirurgião Vascular Assistente do Instituto Dante Pazzanese de Cardiologia - São Paulo (SP), Brasil.

${ }^{2}$ Doutor; Cirurgião Vascular Chefe do Instituto Dante Pazzanese de Cardiologia - São Paulo (SP), Brasil.

${ }^{3}$ Cirurgião Vascular Assistente do Instituto Dante Pazzanese de Cardiologia - São Paulo (SP), Brasil.

${ }^{4}$ Residente em Cirurgia Vascular do Instituto Dante Pazzanese de Cardiologia - São Paulo (SP), Brasil.

Conflito de interesse: nada a declarar.

Submetido em: 10.02.11 Aceito em: 12.08.11

J Vasc Bras. 2011;10(4):308-311.
}

Background: Hemodialysis access fistula may involve creation of a tunnel for the accommodation of autologous vein or synthetic graft. The route and extent depend on the location of the anastomosis and fistula body.Currently, the tunnelers used are rigid metallic cylindrical structures. Various sizes

Methods: This is a pilot study. The tunneler developed consists of a cylindrical structure composed of stainless steel wire with a handle at the proximal end and dual interchangeable conical tip at the distal end. It is covered with a cylindrical sheath of polyethylene. Its technical surgical application and

Results: Characteristics of flexibility and conformability made possible the same mini tunnel maker to be used in various types of fistulas performed. The tapered distal tip with double diameter, interchangeable head, allowed the same apparatus to be used in autogenos and graft fistulas surgical

Conclusion: The same mini malleable tunneler was used in all fistulas, regardless of the site of anastomosis and type of fistula performed without 


\section{Introdução}

A cirurgia de confecção de fístula arteriovenosa para hemodiálise pode envolver a criação de um túnel para a acomodação do enxerto vascular, seja ele realizado com a veia autógena ou prótese sintética. O seu trajeto e extensão dependem do local escolhido para a realização da fístula.

$\mathrm{Na}$ atualidade, os tunelizadores utilizados são estruturas cilíndricas metálicas rígidas e dessa forma podem exigir múltiplas extensões e conformações dos mesmos, e até incisões auxiliares para a acomodação do enxerto.

Isso pode ser um fator de limitação e dificuldade para a confecção destes túneis, e pode predispor o paciente a lesões iatrogênicas e complicações ${ }^{1,2}$. Em estudo prévio, verificamos as vantagens da utilização de um tunelizador maleável na cirurgia de revascularização de membros isquêmicos ${ }^{3}$.

Trata-se de um estudo piloto e o objetivo foi testar a utilização de um mini tunelizador universal metálico e moldável na cirurgia de confecção de fístulas para hemodiálise.

\section{Método}

O tunelizador metálico, maleável, moldável e universal foi desenvolvido no laboratório de Bioengenharia do Instituto Dante Pazzanese de Cardiologia e consiste de uma estrutura cilíndrica híbrida e modular composta de: (1) fio de aço inoxidável maleável e moldável interno, com manopla em extremidade proximal e dupla ponta cônica intercambiável em extremidade distal; (2) bainha cilíndrica externa de polietileno transparente (Figura 1).

Durante a idealização do dispositivo foram perseguidas características como uma liga metálica que oferecesse um equilíbrio entre a dureza, maleabilidade e deformidade necessários, para que fosse possível estabelecermos vários tipos de curva, e utilizarmos o mesmo tunelizador, independentemente do tipo de fístula realizada. Sua dupla ponta cônica intercambiável torna possível a utilização do mesmo modelo em fístulas realizadas com prótese ou veia (Figura 2).

Após aprovação do trabalho pelo Comitê de Ética em Pesquisa (CEP), o mesmo mini tunelizador universal desenvolvido foi utilizado em diversos tipos de fístulas realizadas pelo investigador principal (Figura 3) e observamos a frequência de ocorrência de complicações perioperatórias precoces (30 dias), bem como a perviedade da fístula neste mesmo período.

\section{Resultado}

A experiência inicial obtida com o mini tunelizador idealizado foi obtida nas seguintes fístulas realizadas no período de setembro de 2009 a novembro de 2010, pelo autor principal (Tabela 1).

As características de maleabilidade e capacidade de deformação permitiram a adaptação e utilização do mesmo tunelizador nas diversas fístulas realizadas. A ponta cônica distal com duplo diâmetro, intercambiável, permitiu que o mesmo aparato pudesse ser utilizado na confecção de fístula arteriovenosa realizada com veia autógena ou prótese sintética.

O índice de complicações foi baixo (2\%). Houve um caso de infecção superficial de ferida operatória tratada

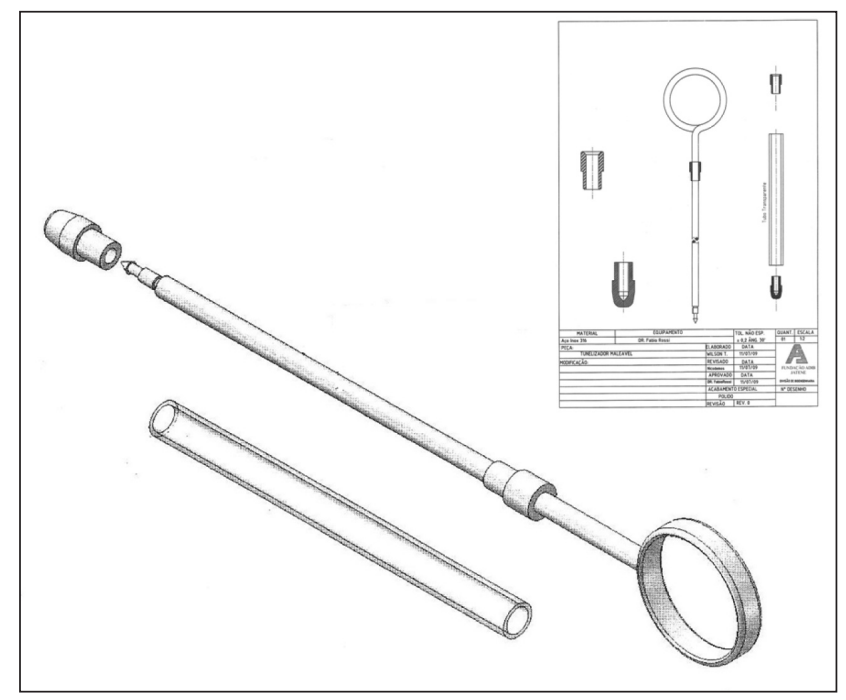

Figura 1. Desenho industrial do mini tunelizador universal.

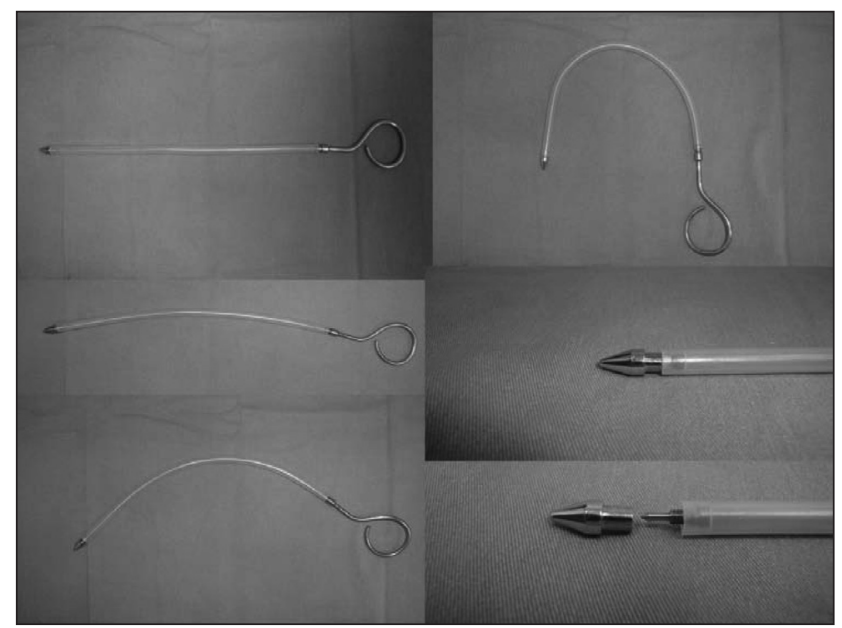

Figura 2. Mini tunelizador universal em múltiplas curvaturas e dupla ponta cônica intercambiável. 


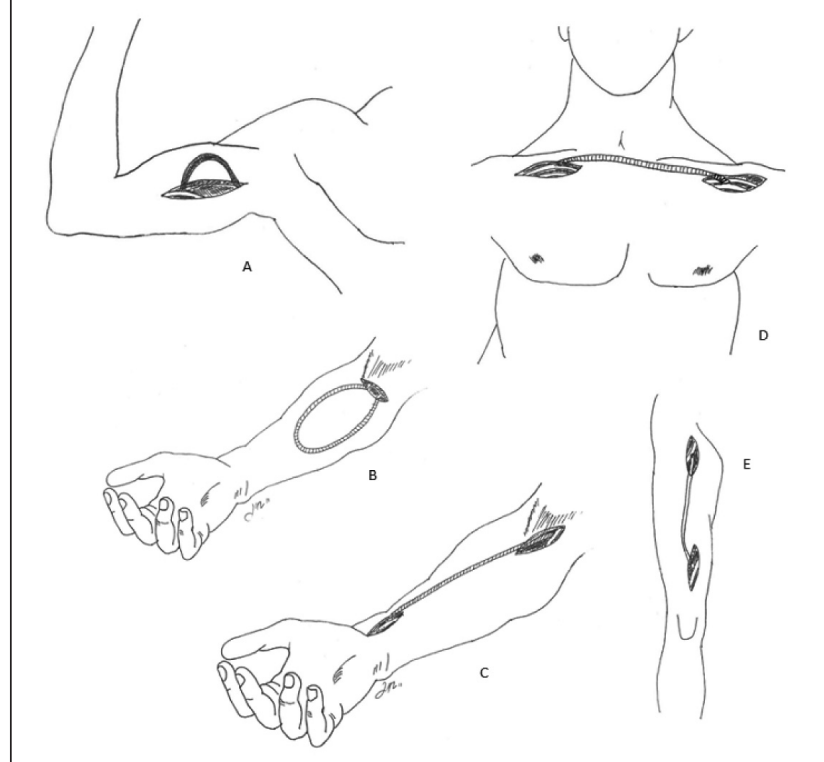

Figura 3. Exemplos de tipos de fístulas realizadas com o mesmo mini tunelizador universal: (A) Transposição de veia basílica; (B) braquiobasílica em alça de PTFE $6 \mathrm{~mm}$; (C) rádio-basílica retilínea com PTFE $6 \mathrm{~mm}$; (D) axilo-axilar retilínea com PTFE $6 \mathrm{~mm}$; (E) safeno-femoral superficial.

com antibiótico oral e um hematoma superficial observado clinicamente. Com um período médio de acompanhamento de 9,9 meses (mínimo 2,0; máximo 16,0 meses) o índice de perviedade foi de $100 \%$.

\section{Discussão}

A hemodiálise é uma forma de tratamento relativamente bem tolerada pelo paciente portador de insuficiência renal crônica (IRC) terminal. Estima-se que nos Estados Unidos (EUA) existam 373 e na Europa 171 pacientes para cada milhão de habitantes realizando esse tratamento ${ }^{4}$. Aproximadamente $40 \%$ da verba gasta pelo Medcare, ou seja, US\$ 6 bilhões são gastos anualmente com esses pacientes 5 .

No Brasil, segundo dados do DATASUS o número de pacientes que desenvolvem IRC terminal vem aumentando. Em 2008, existiam 87 mil pacientes realizando hemodiálise, um aumento de $87 \%$ em relação ao ano de 2000.

Para ingressar em um programa de tratamento é imprescindível a presença de uma fístula em boas condições de uso. A falência e as complicações das mesmas são os principais fatores de internação e custo (nos EUA, US\$ 500.000/ano) nos pacientes em programa de hemodiálise ${ }^{5,6}$.

A infecção e a necrose da ferida operatória são as complicações precoces mais frequentes ${ }^{7,8}$ e a tunelização do enxerto pode ser um fator importante na ocorrência desse
Tabela 1. Fístulas arteriovenosas realizadas com o mini tunelizador universal e complicações observadas em até 30 dias de pós-operatório.

\begin{tabular}{|c|c|c|c|}
\hline Paciente & Idade & Fístula & Complicação \\
\hline 1 & 27 & $\begin{array}{l}\text { Arteriovenosa braquio-basílica em } \\
\text { alça de PTFE } 6 \mathrm{~mm} \text { em antebraço }\end{array}$ & - \\
\hline 2 & 34 & Transposição deveia basílica & - \\
\hline 3 & 65 & $\begin{array}{l}\text { Arteriovenosa safeno-femoral } \\
\text { comum }\end{array}$ & - \\
\hline 4 & 36 & Transposição deveia basílica & - \\
\hline 5 & 70 & $\begin{array}{l}\text { Arteriovenosa braquio-axilar } \\
\text { retilínea de PTFE } 6 \mathrm{~mm}\end{array}$ & - \\
\hline 6 & 77 & $\begin{array}{l}\text { Transposição de } \\
\text { veia basílica }\end{array}$ & $\begin{array}{l}\text { Infecção } \\
\text { superficial de } \\
\text { ferida operatória }\end{array}$ \\
\hline 7 & 25 & $\begin{array}{l}\text { Arteriovenosa safeno-femoral } \\
\text { superficial }\end{array}$ & \\
\hline 8 & 89 & $\begin{array}{l}\text { Arteriovenosa axilo-axilar em } \\
\text { ponte retilínea de PTFE } 8 \mathrm{~mm}\end{array}$ & - \\
\hline 9 & 56 & $\begin{array}{l}\text { Transposição de } \\
\text { veia basílica }\end{array}$ & - \\
\hline 10 & 67 & $\begin{array}{l}\text { Arteriovenosa safeno femoral } \\
\text { superficial }\end{array}$ & $\begin{array}{l}\text { Hematoma } \\
\text { superficial }\end{array}$ \\
\hline 11 & 72 & $\begin{array}{l}\text { Arteriovenosa rádio-basílica } \\
\text { retilínea com PTFE } 6 \text { mm em } \\
\text { antebraço }\end{array}$ & - \\
\hline
\end{tabular}

tipo de complicação. É bem sabido que a fístula não deve ser posicionada muito profundamente, pois pode dificultar a punção da mesma, nem muito superficialmente, pois pode aumentar o risco de necrose e infecção da ferida operatória. O número de incisões e de dissecção cirúrgica necessárias durante a tunelização do enxerto podem influenciar a incidência de infecção'. A utilização do equipamento aqui apresentado pode diminuir o número de incisões necessárias e provavelmente a frequência de infecção.

O local ${ }^{9}$, configuração ${ }^{10}$ e material ${ }^{11}$ utilizado para a realização da via de acesso são fatores que podem influenciar a frequência de complicações.

As indicações para hemodiálise têm sido estendidas. Os pacientes são cada vez mais idosos, diabéticos, imunodeprimidos, clinicamente instáveis e muitas vezes submetidos a múltiplos acessos anteriores, aumentando a complexidade da cirurgia.

Não é infrequente a realização de tunelização do enxerto em posições extra-anatômicas, com próteses em longos trajetos e até mesmo utilizando-se parcialmente fístulas previamente realizadas.

Em nossa casuística, em dois pacientes foram confeccionadas novas fístulas utilizando-se parcialmente trajetos fistulosos realizados anteriormente. No primeiro, o 
paciente desenvolveu um pseudoaneurisma infectado em segmento médio, de transposição de veia basílica realizada anteriormente. Após drenagem, desbridamento e isolamento do local, foi realizado novo trajeto fistuloso com PTFE 6 $\mathrm{mm}$, entre o segmento remanescente de veia basílica anastomosada em artéria braquial e veia axilar, pois o mesmo não possuía mais veia autógena no membro reoperado. No segundo paciente, uma fístula realizada com a veia safena em artéria femoral superficial distal evoluiu com obstrução precoce, e novo trajeto fistuloso em alça foi estabelecido com a realização de anastomose com a artéria femoral comum ipsilateral.

A utilização de tunelizadores rígidos pré-moldados em curvaturas pré-determinadas, existentes na atualidade, pode limitar sua adaptação ou até mesmo causar lesões de estruturas e órgãos presentes em trajetos fistulosos alternativos.

Não existe na literatura descrição de modelo de tunelizador moldável semelhante ao aqui apresentado.

Nossa experiência inicial na cirurgia de confecção de fístulas para hemodiálise, após aquela obtida com o mesmo conceito de tunelizador moldável e maleável na cirurgia de revascularização de membros inferiores isquêmicos ${ }^{3}$, demonstra apenas dois casos de complicações leves (infecção superficial, hematoma) tratadas conservadoramente.

Esses resultados, somados à facilidade de manuseio, baixo custo e versatilidade obtida com o emprego do mini tunelizador universal, indica que o mesmo pode substituir com vantagens aqueles rígidos utilizados na atualidade.

\section{Conclusão}

Os dados sugerem que o mini tunelizador metálico maleável e moldável pode ser utilizado com segurança e vantagens nas cirurgias de confecção de fístula para hemodiálise. Sua versatilidade e baixo custo de produção podem levá-lo a substituir os tunelizadores rígidos utilizados na atualidade e apontam para estudos mais elaborados e com casuística adequada para avaliação do real valor do novo instrumento.

\section{Referências}

1. Blumenberg RM, Gelfand ML. A simple and inexpensive tunneler for use in peripheral vascular surgery. Surgery. 1974;75(2):305-7.

2. Parsonnet $\mathrm{V}$, Driller J. A tunneler for bypass vascular surgery. Arch Surg. 1973;106(2):236-7.

3. Rossi FH, Izukawa NM, Oliveira LA, et al. Tunelizador maleável moldável universal na cirurgia de revascularização do membro inferior isquêmico. J Vasc Bras. 2010;9(1):21-4.

4. Marx AB, Landmann J, Harder FH. Surgery for vascular access. Curr Probl Surg. 1990;27(1):1-48.

5. Feldman HI, Held PJ, Hutchinson JT, et al. Hemodialysis vascular access morbidity in the United States. Kidney Int. 1993;43(5):1091-6.

6. Aman LC, Levin NW, Smith DW. Hemodialysis access site morbidity. Proc Clin Dial Transplant Forum. 1980;10:277-84.

7. Dobkin JF, Miller MH, Steigbigel NH. Septicemia in patients on chronic hemodialysis. Ann Intern Med. 1978;88(1):28-33.

8. Yu VL, Goetz A, Wagener M, et al. Staphylococcus aureus nasal carriage and infection in patients on hemodialysis. Efficacy of antibiotic prophylaxis. N Engl J Med. 1986;315(2):91-6.

9. Munda R, First MR, Alexander JW, et al. Polytetrafluoroethylene graft survival in hemodialysis. JAMA. 1983;249(2):219-22.

10. Kester RC. Arteriovenous grafts for vascular access in haemodialysis. Br J Surg. 1979;66(1):23-8.

11. Schuman ES, Gross GF, Hayes JF, et al. Long-term patency of polytetrafluoroethylene graft fistulas. Am J Surg. 1988; 155(5):644-6.

Correspondência Fabio H Rossi

Departamento de Cirurgia Vascular Instituto Dante Pazzanese de Cardiologia Av Dr. Dante Pazzanese, 500 - Ibirapuera CEP: 04012-180 - São Paulo (SP), Brasil E-mail:vascular369@hotmail.com

Contribuições dos autores Concepção e desenho do estudo: FHR Análise e interpretação dos dados: FHR Coleta de dados: FHR, GSL,PBM, FMA, ESJ, BLA Redação do artigo: FHR Revisão crítica do texto: NMI, AKP Aprovação final do artigo*: FHR, NMI, AKP, GSL, PBM, FMA, ESJ, BLA Responsabilidade geral pelo estudo: FHR Informações sobre financiamento: FHR *Todos os autores leram e aprovaram a versão final submetida ao J Vasc Bras. 